Research Article

\title{
Effect of Enzymes Action on Crushing Strength of Sunflower Kernels to Compressive Loading
}

\author{
E. Danso-Boateng *
}

Department of Chemical Engineering, Kumasi Polytechnic, P. O. Box 854, Kumasi, Ghana

Received 22 November 2010; Revised 7 June 2011; Accepted 30 January 2011

\begin{abstract}
The crushing strength of enzyme-treated and untreated sunflower kernels were measured in terms of compressive force and deformation at cracked point using Lloyd Tensometer provided with a $500 \mathrm{~N}$ compression load cell. This was done to determine the effect of enzyme action on sunflower kernel cell-wall structure, the extent of the cell-wall disruption by the enzymes, and how this could affect oil extraction systems. Four varieties of cell-wall degrading enzyme preparations were used. Deformation values for all the enzyme treated kernels were found to be lower than those for untreated kernels under both horizontal and vertical kernel loading positions. The enzyme treated kernels required lower compressive force to crush than the untreated kernels in all loading positions. Among the various enzymes, 'Viscozyme' treated kernels gave the lowest force to initiate crack. Thus, the extent of kernel cell-wall disruption was found to depend on the type of enzyme used.
\end{abstract}

Keywords: Crushing strength, enzymes treatment, sunflower kernel, compressive force, deformation.

\section{Introduction}

Sunflower (Helianthus annuus L.) seed is a very essential oilseed crop due to its high oil content and relatively high nutritious value of the oil [1]. The seed comprises of between $44-51 \%$ oil content, $20-22 \%$ of the hull, $17-$ $19 \%$ protein, $15-20 \%$ fibre and $0.4 \%$ ash, depending on the variety [2]. The most widely and/or presently used technique for extracting oil from sunflower seeds is by the conventional method, which involves dehulling, grinding and heat treatment to break down the oil-bearing cells, followed by mechanical pressing [3]. The conventional method is however unable to extract all of the oil from sunflower seeds. Some of the oil bodies, however, often remained strongly bonded to the cell matrix and require extensive treatment in order to achieve efficient separation. Some studies have reported that enzymes can enhance the efficiency of oil extraction rate by weakening the oilseed cell-wall structure, and therefore improving diffusivity of the cell-wall to oil passage $[4,5]$. However, a measure of the extent of the cell-wall degradation by enzyme action remains a challenge as little is known about this important feature.

The extent of the effect of enzyme action on the seed cell-wall matrix could be well characterised by the mechanical properties of the seed kernel in terms of its crushing properties under compressive loading. Having information on this feature will be vital for a feasible design of oil extraction systems and the optimisation of the

\footnotetext{
*E-mail address: edansoboateng@yahoo.com

ISSN: 1791-2377 @ 2009 Kavala Institute of Technology. All rights reserved.
}

extraction process parameters. Reports on the crushing characteristics of sunflower seed and many other oilseeds are available in literature, but very little is known about enzyme treated sunflower seed or kernel (dehulled seed).

Fracture properties of an oilseed were first reported by Bilanski [6], who measured the force and energy required for soy bean coat to initiate crack at different moisture contents. When the seed moisture content increased from 1 to $16 \%$, the average force to initiate crack for compression of soy bean with the hilum in the horizontal loading position reduced from 57.8 to 44.4 N. Saiedirad et al. [7] performed similar tests with cumin seeds, by measuring the average compressive force, seed rupture force and energy absorbed at different moisture contents under quasi-static loading conditions for both horizontal and vertical orientations. Their work indicated that cumin seeds are more flexible in horizontal orientation; and that the force required for initiating rupture decreased whilst the energy absorbed increased with increase in moisture content for vertical and horizontal orientations. Similar observations have been reported for other seeds, for example hazelnut [8], fennel seeds [9], barley grains [10], pine nuts [11, 12], and Jatropha curcas L. [13].

Knowledge on cracking characteristics of melon seeds has also been reported. These seeds have similar shape to that of sunflower seeds. Paksoy et al. [14] performed tests on the mineral content and some physical properties of watermelon seeds as functions of moisture content. Their results showed that the rupture force decreased as moisture content of the seeds increased. Razavi \& Milani [15] also made similar observations on their study with watermelon. 
Cracking characteristics of sunflower whole seeds (undehulled seeds) and kernels were reported by Khodabakhshian et al. [16]. They measured the mechanical parameters such as force, deformation and energy absorbed for fracturing of sunflower seeds and kernels at different moisture contents between 3 and $14 \%$ dry basis, under vertical and horizontal orientations. The results showed that rupture forces for both sunflower seed and kernel decreased with increasing moisture content, while in both loading orientations and for all varieties and size categories, the absorbed energy and deformation at rupture point increased as moisture content increased.

The studies on various seeds, so far, concerned with the effect of moisture content on crushing and characteristics of the seeds. These revealed that loading position and seed or kernel moisture content have significant effect on the force required to initiate crack as well as cause deformation. However, this type of information has not been reported for sunflower seeds or kernels treated with enzymes. The purpose of this study was to analyse the possibility of weakening sunflower kernels (dehulled seeds) by the use of hydrolytic and cell-wall degrading enzymes such as cellulases, pectinases and carbohydrases by analysing the mechanical characteristics of the kernels. It is anticipated that the results from these studies would throw more light on the extent of enzyme action on sunflower kernel cell-wall structure and how this could reduce energy usage and improve oil flow in extraction systems.

\section{Materials and Methods}

The sunflower (Helianthus annuus L.) kernels (dehulled seeds) were obtained from Healthy Living, Loughborough, UK. The kernels were manually sorted out to remove foreign materials such as dirt, stones, as well as broken and undeveloped kernels. The enzyme preparations; 'Viscozyme' L and 'Ultraflo' L, were obtained from Novozymes (Bagscaerd, Denmark), whilst 'Ronozyme' L and 'Roxazyme' G2 were from Roche (Basel, Switzerland). These enzymes, referred to as 'commercial enzymes', were supplied as aqueous solutions.

The initial moisture content of the kernels were determined using the ISA3576 [18] standard hot air method at a temperature of $105 \pm 1^{\circ} \mathrm{C}$ for 24 hours.

Ten millilitres $(10 \mathrm{ml})$ of each enzyme preparation was measured and added to $20 \mathrm{~g}$ kernels in separate beakers already containing $100 \mathrm{ml}$ of citrate buffer ( $\mathrm{pH} \mathrm{5.0)}$ and placed in a water bath at $50^{\circ} \mathrm{C}$ for $24 \mathrm{hrs}$. The liquid portions of each sample were then discharged and the kernels transferred into beakers, covered tightly and placed in a dessicator. The enzyme treated kernels were then taken from the dessicator and dried in an oven at $105 \pm 1{ }^{\circ} \mathrm{C}$ for 50 minutes to maintain the moisture content at $4.25 \%$ dry basis before the tests. This was done to ensure that the moisture content of the enzyme treated kernels were the same as the raw kernels. The samples were then placed in a dessicator to cool and also to prevent the kernels from losing moisture. The kernels were taken straight from the dessicator and tested.

The initial test was to determine the compressive force and deformation for untreated (raw) sunflower kernels under both vertical and horizontal loading positions. This was performed on the biggest kernels (4-5 x 12-14 mm: B x L) and smallest kernels (3-5 x 7-9 mm: B x L).

The compression tests were performed with a "Lloyd

Tensometer" (Lloyd instruments, L-10000 Tensometer, UK) provided with a $500 \mathrm{~N}$ compression load cell and an integrator. A sketch of the experimental set-up is shown in Fig.1.

(a)

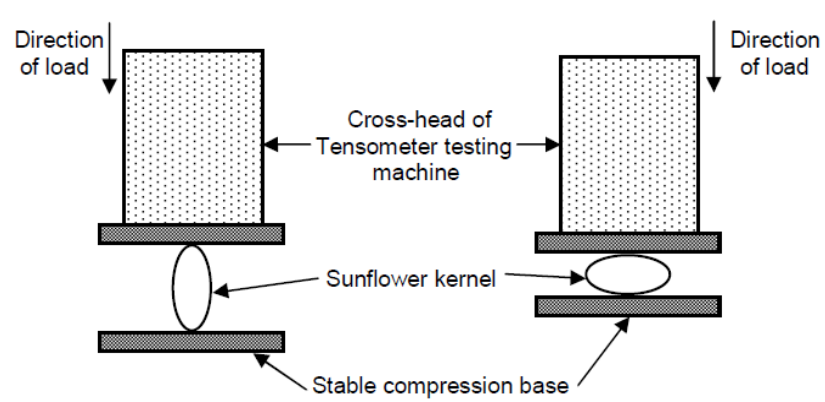

Fig. 1. Sketch of experimental set-up for compression test of sunflower kernels under different loading positions using Lloyd Tensometer: (a) vertical position, (b) horizontal position.

During the test, each kernel was placed between two parallel plates of the Tensometer (see Fig. 1) and compressed at a fix cross-head speed of $3.0 \mathrm{~mm} / \mathrm{min}$ until the seed kernel cracked. The compression test was stopped as soon as the cracked point was detected by the Tensometer, and the force values and their corresponding deformation values recorded directly from the Tensometer. These were detected from the characteristics of the forcedeformation curve generated by the Tensometer. In most cases, the cracked point was visually detected as the kernel splitted into halves or cracked. The kernels were positioned in both horizontal surface (longitudinal axis of the kernel normal to the direction of the loading) and vertical surface (longitudinal axis of the kernel parallel to the direction of the loading).

Prior to all the experiments, the kernels were visually examined and those with cracks discarded before loading for the compression test. This ensured that the values obtained represented the true results.

In each experiment, fifty (50) kernels were tested under both loading orientations to give a total of hundred (100). In the experiments which involved compression of the enzymes treated kernels, the kernels were chosen at random and tested without considering their sizes.

During the experiment, images of the untreated (raw) and enzyme treated sunflower kernels were taken using a microscope (Nikon Optiphot OPTIPHOT-2) fitted with a camera (Leica DFC-320) and recorded.

\section{Results and Discussions}

\subsection{Deformation of kernels occurring at cracked point}

The deformation values occurring at cracked point for enzymes treated and untreated (raw) sunflower kernels loaded in both vertical and horizontal orientations are shown in Fig. 2. These deformation values are average values for the 50 samples tested in each compression experiment. The term 'deformation' in this study refers to the size of rupture of the kernel. The average deformation values for large untreated sunflower kernels were found to be a little higher than small untreated kernels under vertical loading position. However, a different trend was obtained when the kernels 
were loaded in the horizontal position. In the latter instance, the average deformation values for large untreated kernels were smaller than the corresponding values for small untreated kernels for horizontal loading (see Fig. 2).

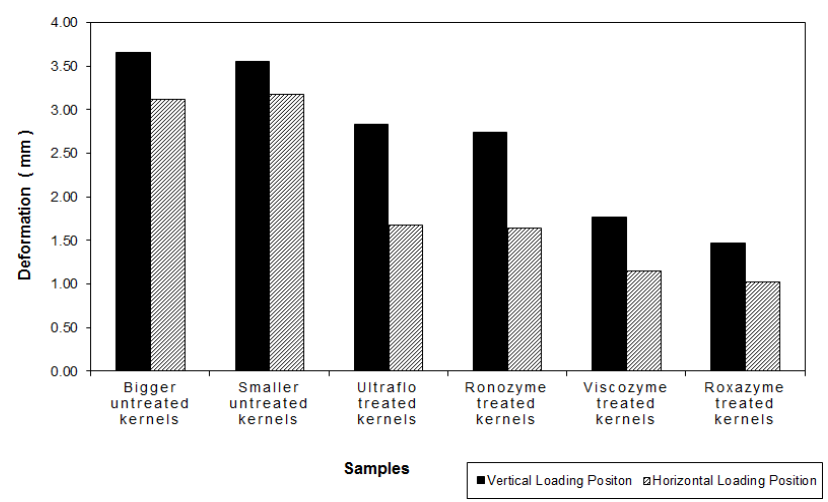

Fig. 2. Graph of variation of deformation values of enzyme treated and untreated sunflower kernels under horizontal and vertical loading orientations.

It was observed that the average deformation values for all kernels loaded in the horizontal orientation were lower than the corresponding values for vertical loading. For the horizontal loading position, the deformation values obtained ranged from $1.02-3.17 \mathrm{~mm}$, whilst values obtained for vertical loading position were between $1.47-3.66 \mathrm{~mm}$. This was because comparatively, sunflower kernels are less deformable at crack point when the kernels were loaded in the horizontal loading position as compared with when the kernels were loaded vertically. This shows that the kernels are less flexible and more resistant to cracking when loaded in the horizontal orientation as compared to the vertical. The structural arrangement of cotyledons in sunflower kernel is shown in Fig. 3 below.
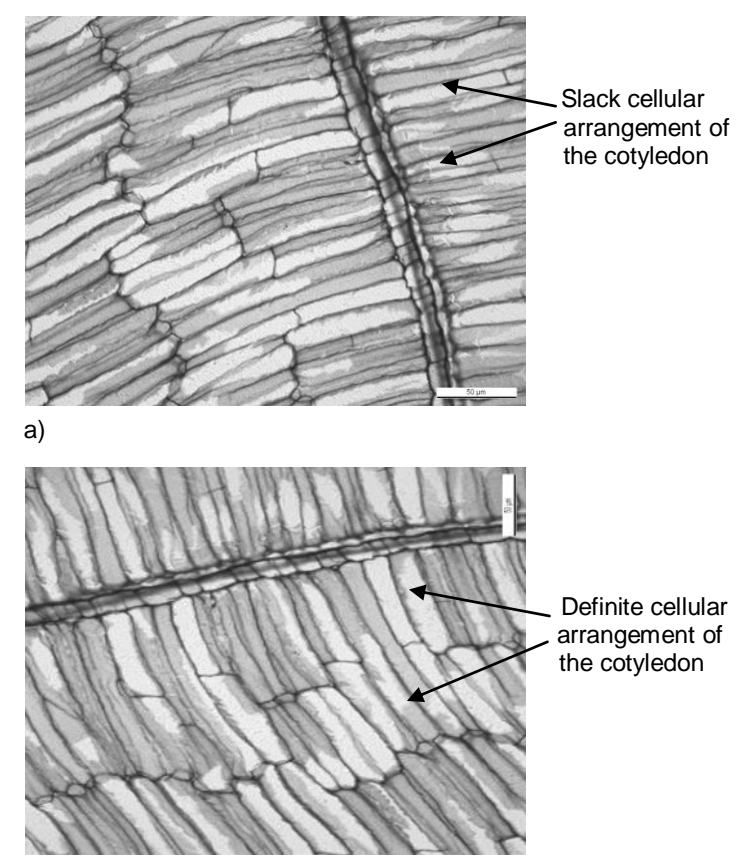

Fig. 3. Microscopic cellular structure of sunflower kernel taken at the middle side of the kernel showing the cotyledon arrangement: (a) vertical orientation, (b) horizontal orientation.
As shown in Fig. 2, the average deformation values for virgin kernels were remarkably higher than all the enzyme treated kernels under both horizontal and vertical orientations of kernel loading. The lower deformation values obtained in the case of the enzyme treated kernels under both loading positions indicates that the enzyme treated kernels are less resistant to cracking, even at lower forces. This shows that the enzymes weakened the kernels cell-wall structure making the cotyledon tissues inflexible. The average values for virgin kernels were between 3.55 - 3.66 $\mathrm{mm}$ under vertical loading and $3.11-3.17 \mathrm{~mm}$ for horizontal loading position. The average values for enzyme treated kernels loaded vertical were between $1.47-2.83 \mathrm{~mm}$, and 1.02 - $1.67 \mathrm{~mm}$ for horizontal loading position. Average values for 'Roxazyme' treated kernels were the lowest as compared with the other enzyme treated kernels. The order was Roxazyme < Viscozyme < Ronozyme < Ultraflo, for both orientations of kernel loading.

\subsection{Compressive Force}

Results obtained for force required to cause rupture of enzymes treated and untreated (raw) sunflower kernels under compressive loading in vertical and horizontal orientations are shown in Fig. 4 below. The values used are average force values for the 50 kernels tested in each experiment. The graph shows variation of force to crush the various enzymes treated kernels and untreated kernels. It was observed that values of force required to crack the various enzyme treated kernels were considerably lower than those obtained for the untreated kernels for both vertical and horizontal orientations of loading. Again, this results shows that the enzymes weakened the sunflower kernel cell-wall structure.

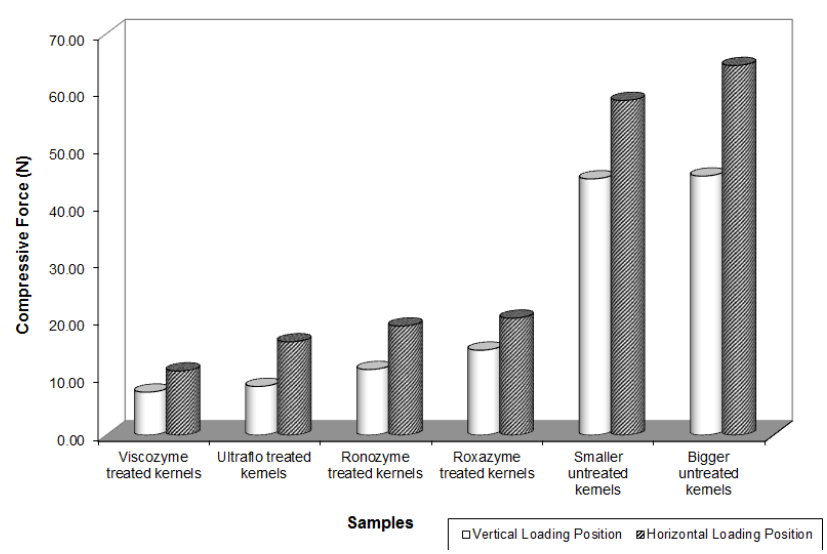

Fig. 4. Variation of force to initiate crack for enzyme treated and untreated sunflower kernels under vertical and horizontal loading orientations.

As a result, the cotyledon tissues of the kernels were soft (this is shown in Fig.5 below). This meant that an even lower compressive force applied is enough to initiate crack as compared to the virgin kernels. For the small untreated (raw) kernels, the average forces required were 44.69 and $58.38 \mathrm{~N}$ for vertical and horizontal loading positions respectively. The large untreated kernels required 45.19 and $64.47 \mathrm{~N}$ for vertical and horizontal loading orientations respectively.

For the various enzyme treated kernels, the force required to crush 'Viscozyme' treated kernels were the 
lowest for both vertical and horizontal loading orientations. The order was as follows: Viscozyme < Ultraflo < Ronozyme < Roxazyme, with values of 7.50, 8.49, 11.45 and $14.82 \mathrm{~N}$ - for vertical loading orientation, and 11.20, $16.28,19.03$ and $20.40 \mathrm{~N}$ - for horizontal loading orientation respectively. The lower force required for 'Visozyme' treated kernels than the other enzymes might be due to Viscozyme's activity on branched pectin substances, which is able to break down the kernel cotyledon cell-wall structure better. This indicates that the type of enzyme applied will determine the extent of the kernel cell-wall break down or disruption.

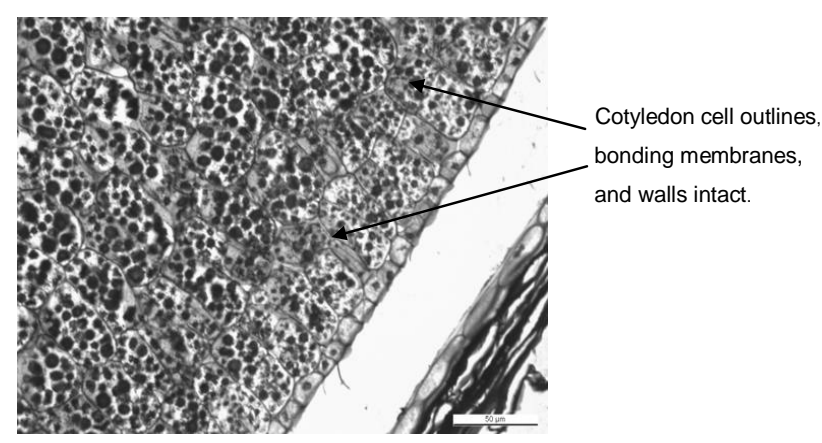

a)

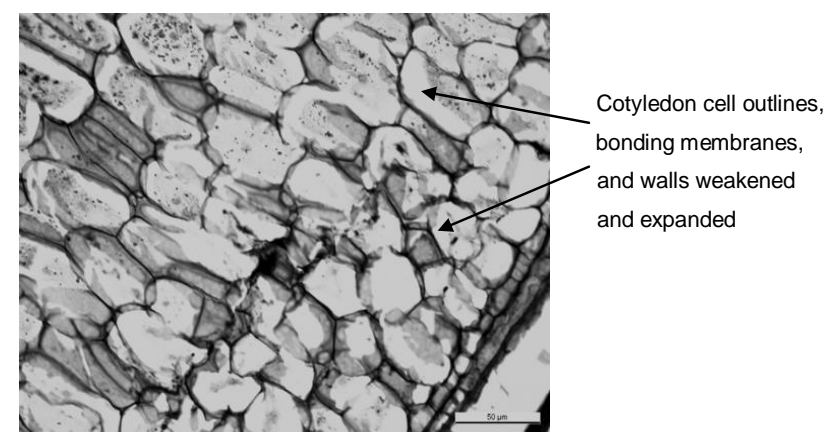

b)

Fig. 5. Microscopic structure of sunflower kernel showing the internal tissues of the cotyledon taken at the extreme side of the kernel: (a) raw kernel, (b) enzyme treated kernel.

The average maximum force values obtained for 'Viscozyme' treated kernels were lower than the smallest values obtained by Khodabakhshian et al. [16] for moisture laden sunflower kernels in both orientations. The low values of rupture force for sunflower kernels obtained by Khodabakhshian et al. [16], namely, $11.64 \mathrm{~N}$ for vertical loading and $16.94 \mathrm{~N}$ for horizontal loading orientation were obtained at a kernel moisture content of $14 \%$ dry basis. However, when the kernel moisture content is too high oil extraction is not favourable [17]. Therefore, it is imply that with the lower values of compressive force obtained for 'Voscozyme' treated sunflower kernels $(7.50 \mathrm{~N}$ for vertical and $11.20 \mathrm{~N}$ for horizontal) at moisture content of $4.25 \%$ dry basis, lesser energy will be required to crush
'Voscozyme' treated sunflower kernels in extraction systems.

All the values obtained indicated that sunflower kernels loaded vertically required lower force to initiate crack than when loaded in the horizontal orientation. This was because the applied external forces created shear stresses in the internal tissues which caused crack of the cotyledon as the kernels were loaded vertically compared to the definite cellular matrix in the cotyledon cell-wall structure when loaded horizontally. The structure of the internal tissues of sunflower kernel cotyledon is shown in Fig. 3, in Section 3.1. Mostly, force at rupture was easily observed visually as kernel cotyledons crack into halves for both loading positions.

Force-deformation characteristics were observed during the experimental study. This is shown in Fig. 6 below. For all the sunflower kernels (untreated and enzyme treated) tested, the compressive force on the kernel increased with an increased in deformation. However, there was a decrease in the force after crack occurred in the kernel, but the applied force to the kernel increased with further increased in deformation. The force beyond the crack point was not considered essential since it does not correspond to the force required to crush the kernel after crack had occurred.

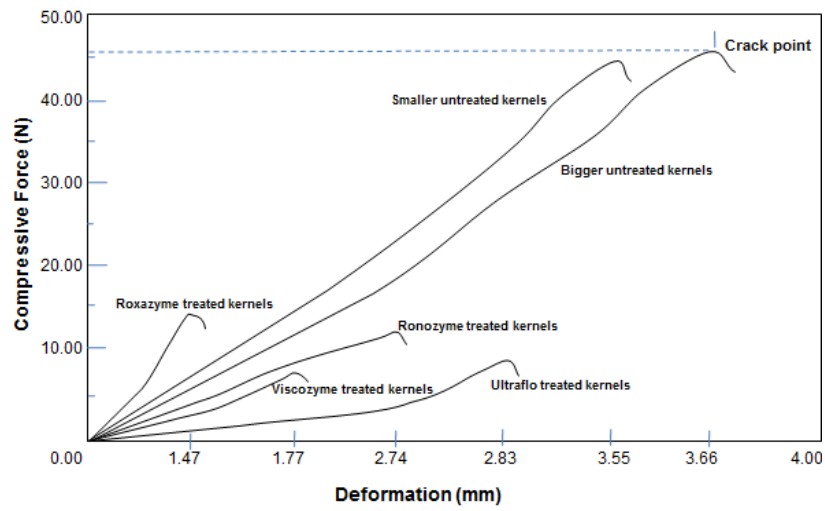

Fig. 6. Force-deformation characteristics for enzymes treated and untreated sunflower kernels deformed at $3.0 \mathrm{~mm} / \mathrm{min}$ under vertical loading position.

\section{Conclusion}

In this work, crushing force and deformation for untreated and enzyme treated sunflower kernels were examined. The force required to initiate crack for enzymes treated sunflower kernels were lower than that required for untreated kernels irrespective of the orientation of the kernel to the applied compressive load.

Among the enzymes studied, it was observed that the force required to initiate crack, and the extent of kernel cell-wall disruption depend on the type of enzyme used.

\section{References}

1. N. Singh, R. Singh, K. Kaur, and H. Singh, J.Food Chem. 66, 241 (1999).

2. F. D. Gunstone, Vegetable oils in food technology: Composition, Properties and Uses, Blackwell Publishing, Oxford, pp. 128-145
(2002).

3. M. Marcotte, S. Grabowski, D. I. M. LeBlanc, and K. Darley, Separation technologies, R \& D for the food industry: A Canadian Survey, pp. 12-39 (2002). 
4. K. A. Campbell, Iowa State University, DAI-B 71/05, 179 (2010). 5. S. Latif, F. Anwar, and M. Ashraf, J.Food Lipid 14, 424 (2007).

6. W. K. Bilanski, Trans.ASAE. 9, 360 (1966).

7. M. H. Saiedirad, A. Tabatabaeefar, A. Borghei, M. Mirsalehi, F. Badii, and M. Ghasemi Varnamkhasti, J.Food Engng. 86 (4), 565 (2008).

8. F. Ozdemir, and I. Akinci, J.Food Engng. 63, 341 (2004)

9. H. Ahmadi, K. Mollazade, J. Khorshidi, S. S. Mohtasebi, and A. Rajabipour, J.Agri.Sci. 1, 66 (2009).

10. H. Tavakoli, S. S. Mohtasebi, A. Rajabipour, and M. Tavakoli, Res.Agr.Eng. 55 (3), 85 (2009).

11. K. Vursavuş, and F. Özgüven, Biosystems Engineering 90 (2), 185 (2005).
12. L. M. Cárcel, J. Bon, L. Acuña, I. Nevares, M. del Álamo, and R. Crespo, Int.Conf.Food Innova. 1 (2010).

13. K. Shkelqim, and M. Joachim, Ind.Crops Prod. 32 (2), 129 (2010).

14. M. Paksoy, C. Aydin, Ö. Türkmen, and M. Seymen, Pak. J. Bot. 42 (4), 2775 (2010).

15. S. M. A. Razavi, and E. Milani, African Journal of Agricultural Research, 1 (3), 065 (2006).

16. R. Khodabakhshian, B. Emadi, M. H. Abbaspour Fard, and M. H Saiedirad, Int.J.Food Engng. 7 (3), 19 (2011).

17. A. Kartika, P. Y. Pontalier, and L. Rigal, Ind.Crops Prod. 22, 207 (2005).

18. ISA3576 (1996) 